\title{
The Promise of Locoregional Radiopharmaceutical Therapy: Will It Ever Be Realized?
}

A by Boucher et at. in this issue of The by Boucher et al. in this issue of The Journal of Nuclear Medicine underscores what most of us have accepted as conventional wisdom-that locoregional adjuvant therapy with radiolabeled lipiodol increases disease-free and perhaps overall survival in patients with hepatocellular carcinoma.

These results confirm the results of a prospective study that used lower amounts of ${ }^{131} \mathrm{I}(1,850 \mathrm{MBq}$, in contrast to $2,400 \mathrm{MBq}$ in the Boucher study).

Why, then, is such therapy, either with ${ }^{131}$ I-labeled lipiodol or with ${ }^{188} \mathrm{Re}$ labeled lipiodol, not more widely used? Indeed, why, with the notable exception of ${ }^{131} \mathrm{I}$ for thyroid disorders, is all radiopharmaceutical therapylocoregional or systemic — underused?

Articles in prominent newspapers and magazines have bemoaned the lack of proper use of systemic radiopharmaceutical therapy in lymphoma. We all know that bone pain palliation therapy with radioisotopes, shown to be of considerable utility in trials of excellent design, is also underused. What is the malaise that afflicts us, and what may be done about it?

There is, unfortunately, no clear and simple answer. Regulations that make it difficult to treat with radioactivity? Perhaps, but are these not the same regulations that exist for thyroid disorders, particularly thyroid cancer? "Turf" issues among referring physicians? Again, perhaps, but how would these explain the underuse of radioimmunotherapy in European countries with national health coverage?

COPYRIGHT (C) 2008 by the Society of Nuclear Medicine, Inc.

DOI: 10.2967/jnumed.107.047639

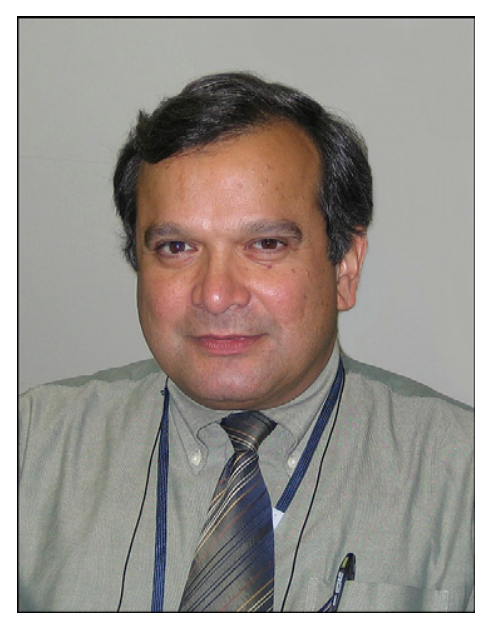

Economics? That is a hard nut to crack. Do we need cost-benefit analyses that are more rigorous than those already available? Admittedly, the field is sparse in this regard, though radioimmunotherapy for lymphoma was approved by the Europeans only after such analyses were considered. (A recent review in this regard is illuminating.)

My own bias is that it is in the marketing. The distrust and fear of radiation are considerable, irrational, and growing. Little attempt is being made to educate the public on the relative risks and benefits of radiation, be it sealed or unsealed, and we have a duty to educate not only our patients but also the lay public, as well as our referring physicians, about the largely theoretic risks associated with radiopharmaceutical therapy. This is central to any resurgence in radiopharmaceutical therapy.

As nuclear medicine physicians, we have increasingly become oriented toward the diagnostic potentials of our specialty and moved away from therapy. The promise of PET is becoming wonderfully evident, and we have em- braced it, and rightly so. We should beware of complacency, though. Radiation oncology is increasingly concerning itself with systemic radiotherapeutics; residents from our radiation oncology program now routinely observe and participate in locoregional and systemic radiopharmaceutical therapy at my institution.

I have little doubt that increasing numbers of radiation oncologists will perform systemic radiopharmaceutical therapy - its promise is evident to them; it has now become more systematically incorporated into their training. Most important, perhaps, is that radiation oncologists have both a patient referral base and a desire to manage patients. That desire may well be diminishing in nuclear medicine physicians, who increasingly practice as diagnosticians; a laudable pursuit, but one that misses out on an equally exciting component of our specialty.

The optimist in me believes that radiopharmaceutical therapy will therefore undergo a resurgence, with its potential being realized by a diverse group of physician scientists-as in the article that is the subject of this perspective. Nuclear medicine has made many seminal contributions to the practice of medicine- ${ }^{131}$ I therapy, nuclear cardiology, PET, and radioimmunotherapy, to name a few-and their crossdisciplinary potential will ensure their continued utility. The practice of radiopharmaceutical therapy will grow, and here's hoping there will be a concomitant growth in the specialty of nuclear medicine. 